\title{
Active multispectral Near-IR detection of small surface targets
}

\author{
Arie N. de Jong, Hans Winkel, Marco J.J. Roos \\ TNO Physics and Electronics Laboratory \\ PO Box 96864, 2509 JG The Hague, The Netherlands \\ E-mail: dejong@fel.tno.nl
}

\begin{abstract}
The detection and identification of small surface targets with Electro-Optical sensors is seriously hampered by ground llutter, leading to false alarms and reduced detection probabilities. Active ground illumination can improve the detection performance of EO sensors compared to passive skylight illumination because of the knowledge of the illumination level and of its temporal stability. Sun and sky cannot provide this due to the weather variability. In addition multispectral sensors with :arefully chosen spectral bands ranging from the visual into the near IR from 400-2500 nm wavelength can take benefit of a variety of cheap active light sources, ranging from lasers to Xenon or halogen lamps. Results are presented, obtained with a wo-color laser scanner with one wavelength in the chlorophyll absorption dip. Another active scanner is described operating at 4 wavebands between 1400 and $2300 \mathrm{~nm}$, using tungsten halogen lamps. Finally a simple TV camera was used with either set of narrow band spectral filters or polarization filters in front of the lamps. The targets consisted of an array of mixed bjects, most of them real mines. The results show great promise in enhancing the detection and identification probabilities f EO sensors against small surface targets.
\end{abstract}

\section{INTRODUCTION}

Active imaging has in principle various advantages compared to passive imaging, which is related to the use of daylight. Daylight is an unreliable source of illumination as the skylight is constantly changing with time due to the variation in cloud and sun position. Moreover the sky brightness distribution is variable, resulting in changes in shadows from vertically itructured objects. Similarly the spectral distribution is dependent on the sky condition. Finally the absolute illumination evel can vary an order of magnitude when cloud cover changes from complete overcast, large solar Zenith angle to clear sky imall solar Zenith angle. For cameras with sensitivity beyond $1.3 \mu \mathrm{m}$ this may lead to conditions with poor signal to noise atio, when the irradiance level comes below $10 \mathrm{~W} / \mathrm{m}^{2} \mu \mathrm{m}$.

Active imaging is a technique, where additional light sources are used. Most of the time, daylight will be excluded to avoid confusion with the reflected artificial light, which means that operation at night is preferred. At TNO-FEL work on active maging started in the early 70's with a scanning laser and a collimator Xenon searchlight [1]. Spectral retro-reflectance data vere collected and the 10 most promising spectral subbands were determined for battlefield surveillance applications.

For detection of surface-laid landmines, active imaging is specially attractive as ranges are relatively short, so one can otentially use low power sources and small sensors such as the generally introduced barcode readers. A principle drawback of the active imaging setup is the dependence of the signal level on the range between the source/receiver and the target. What is basically done however is that the ratio of the signals is used for determining the nature of the object, eliminating the ange effect.

$\mathrm{n}$ this paper a device is described using two lasers as illuminators, colocated with the receiving optics. The laser provides a iny spot on the ground, which is of similar size as grass leaves, thereby producing a lot of vegetation clutter, which is not resent on manmade objects. The spectral bands are chosen below and above the reflectance jump at $700 \mathrm{~nm}$, present in latural vegetation.

$\mathrm{n}$ a second device a scanning radiometer is described with two spectral bands around $2000 \mathrm{~nm}$. In this case standard alogen lamps are used as illuminators, however, they are not colocated with the receivers, so some shadows may be present in one side of the objects. If under control however shade provides information on the shape of the object, so an additional ive [2] can be obtained. The shade effect can be strengthened by using 2 or more lamps, as was done in a laboratory setup at NO-FEL [3].

third device, used with active sources, was a CCD camera with a selected set of interference filters and the same lamp set is used with the scanning radiometer. The spectral bands were selected based upon the reflectance spectra, measured in the 
laboratory with the UV3100 spectrometer on a selection of objects, which may be found in a battlefield with mines (Figure $1)$.

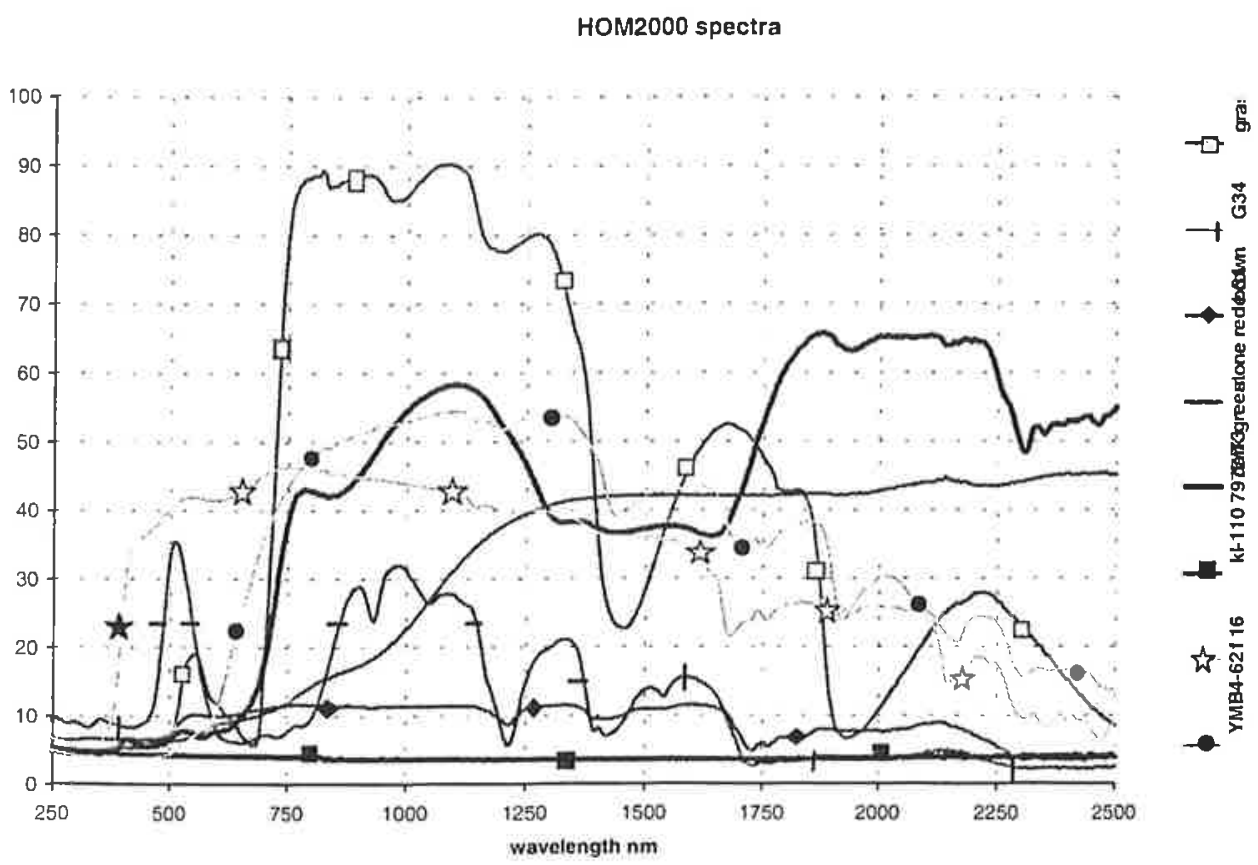

Figure 1: Examples of spectral reflectance curves of a number of materials.

After laboratory testing (described in [3] and [4]), the devices were tested in the field with an arrangement of surface-laid objects, as illustrated in Figure 2, in the TNO mine test facility [5].

The experiments were carried out in the framework of project A00D843 from the Netherlands Defence Research organization. A detailed report on the experiments has been published [6]. In this paper a summary of part of the results is presented.

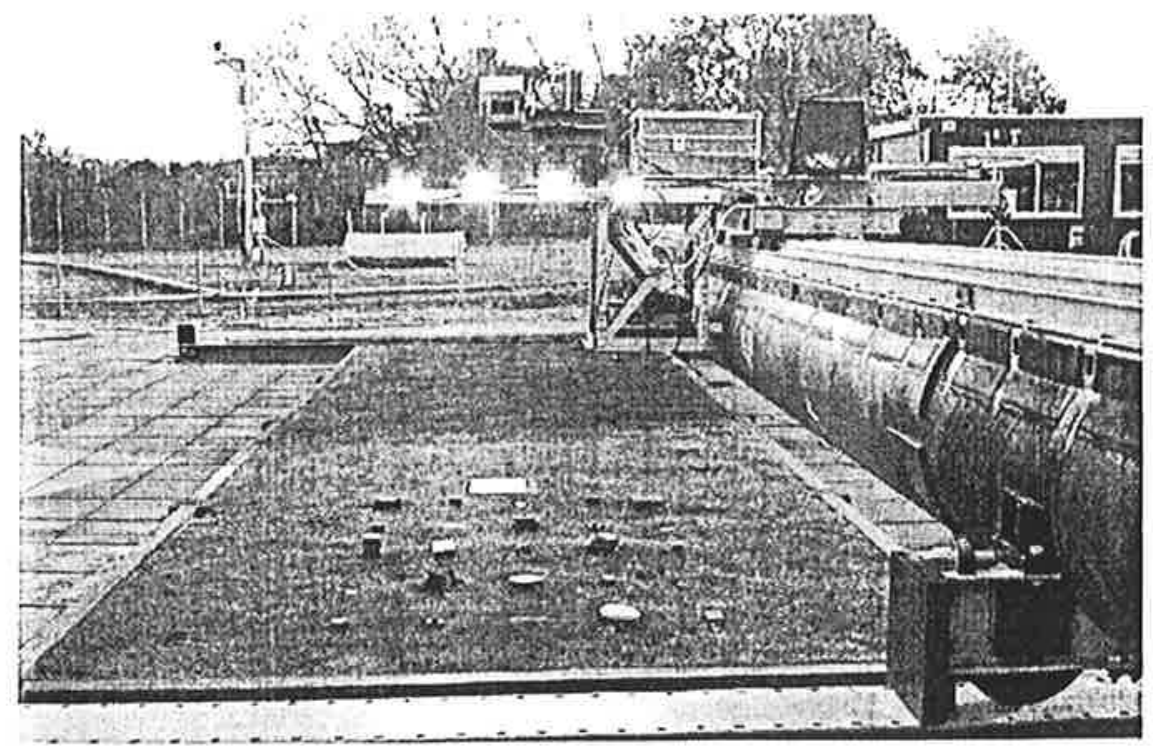

Figure 2: Position of 25 objects in lane 6 with 4 illuminating lamps and SWIR radiometer scanner, looking southward. 


\section{THE TARGETS}

A summary of the most important target characteristics is given in Table 1. In view of the discrimination criteria: color, size, shape, gloss and clutter, relevant data show that some have similar size and shape, but different color; other sets have the same color but different shapes. Some targets have been chosen as they may appear in the field: pieces of wood and stone. All targets were positioned on a grass field in a regular pattern. The numbers $1 \mathrm{~A}$ to $5 \mathrm{E}$ correspond to the object positions in Figure 2 from right bottom to left top, while rows $A$ to $E$ are counted from the right to the left.

The ratio between the reflection coefficients $\rho_{1}$ and $\rho_{2}$ in the chlorophyll dip at $675 \mathrm{~nm}$ and the near IR at $778 \mathrm{~nm}$ shows that all objects are greatly different from the grass background. So the primary use of rationing is for target detection: all nonvegetation objects are easily detected, of course also open soil, which is not taken in the table. The ratio $\rho_{1} / \rho_{2}$ shows however also that we can discriminate a few classes: values between 0.25 and 0.45 and between 0.60 and 0.75 and around 1.00. It is noted however that not only the ratio between the two signals can be considered, but also the absolute signal level, as the source power, the range and the detector responsivity are known. In this way we can discriminate a number of grey levels between black and white.

Table 1: Characteristics of the 25 objects used (an * indicates a real mine).

\begin{tabular}{|c|c|c|c|c|c|c|c|c|}
\hline $\begin{array}{l}\text { Object } \\
\text { no }\end{array}$ & Description & Shape & $\begin{array}{l}\text { Dimensions } \\
\mathrm{L} \times \mathrm{W} \times \mathrm{H} \mathrm{mm}\end{array}$ & Color & $\begin{array}{l}\rho_{1} @ \\
675 \mathrm{~nm}\end{array}$ & $\begin{array}{l}\rho_{2} @ \\
778 \mathrm{~nm}\end{array}$ & $\rho_{1} / \rho_{2}$ & Remarks \\
\hline IA & brown stone & rect $* *$ & $105 \times 55 \times 40$ & brown & 0.100 & 0.134 & 0.75 & \\
\hline $1 \mathrm{~B} *$ & D19 & cyl & $132 \emptyset \times 50$ & black/green & 0.040 & 0.040 & 1.00 & rubber \\
\hline IC & wood (wet) & rect & $120 \times 47 \times 12$ & natural & 0.083 & 0.20 & 0.42 & \\
\hline $1 \mathrm{D} *$ & B38 & cyl & $600 \times 32$ & green/blue & 0.087 & 0.186 & 0.47 & blue side \\
\hline $1 E$ & alu dummy** & cyl & $50 \emptyset \times 50$ & light reen & 0.068 & 0.100 & 0.68 & \\
\hline $2 \mathrm{~A}$ & alu dummy & cyl/disc & $1200 \times 120$ & dark green & 0.123 & 0.43 & 0.29 & \\
\hline $2 \mathrm{~B}$ & alu dummy & cyl & $500 \times 50$ & blue & 0.039 & 0.121 & 0.32 & \\
\hline $2 C^{*}$ & KL1 $107977 / 73$ & cyl & $117 \varnothing \times 45$ & black rubber & 0.040 & 0.040 & 1.00 & \\
\hline $2 \mathrm{D}$ & rusty iron & irreg & $50 \times 100$ curled & brown & 0.100 & 0.100 & 1.00 & \\
\hline $2 \mathrm{E} *$ & butterfly H13 & іпеg & $110 \times 40 \times 10$ & green & 0.087 & 0.085 & 1.02 & \\
\hline $3 \mathrm{~A}$ & alu dummy & cyl & $50 \varnothing \times 50$ & dark green & 0.123 & 0.43 & 0.29 & same as $2 \mathrm{~A}$ \\
\hline $3 \mathrm{~B} *$ & YMB4 62-81 & cyl & $1450 \times 50$ & light brown & 0.30 & 0.46 & 0.65 & \\
\hline $3 \mathrm{C}$ & alu dummy & cyl & $60 \emptyset$ & dark green & 0.123 & 0.43 & 0.29 & same as $2 \mathrm{~A}$ \\
\hline $3 D *$ & box KL110 7976/14 & rect & $149 \times 74 \times 40$ & green & 0.087 & 0.085 & 1.02 & \\
\hline $3 \mathrm{E}^{*}$ & ҮИПІСМ- 1 & cyl & $750 \times 110$ & red/brown & 0.076 & 0.072 & 1.06 & \\
\hline $4 A *$ & butterfly G34 & irreg & $110 \times 40 \times 10$ & light green & 0.064 & 0.085 & 0.75 & \\
\hline $4 \mathrm{~B} *$ & E31 & cyl & $55 \varnothing \times 31$ & green & 0.107 & 0.116 & 0.92 & green side \\
\hline $4 C^{*}$ & UMWP-62-86 & cyl & $126 \times 60$ & brown & 0.30 & 0.46 & 0.65 & same as $3 \mathrm{~B}$ \\
\hline $4 \mathrm{D} *$ & F16 (PVC) & cyl & $560 \times 42$ & grey & 0.44 & 0.46 & 0.96 & \\
\hline $4 \mathrm{E}$ & alu dummy & cyl & $50 \emptyset \times 50$ & red & 0.46 & 0.51 & 0.90 & \\
\hline $5 \mathrm{~A}$ & concrete tile & rect. & $200 \times 150 \times 50$ & grey & 0.082 & 0.134 & 0.61 & \\
\hline $5 \mathrm{~B} *$ & KL110 7982/90 & cyl & $560 \times 42$ & green & 0.087 & 0.085 & 1.02 & \\
\hline $5 C^{*}$ & butterfly H13 & irreg & $110 \times 40 \times 10$ & green & 0.087 & 0.085 & 1.02 & \\
\hline 5D & alu dummy & cube & $50 \times 50 \times 50$ & dark green & 0.123 & 0.43 & 0.29 & same as $2 \mathrm{~A}$ \\
\hline $5 \mathrm{E} *$ & PPM2 01-97 & cyl & $120 \varnothing \times 60$ & black plastic & 0.025 & 0.025 & 1.0 & \\
\hline & grass & irreg & & green & 0.056 & 0.87 & 0.064 & \\
\hline
\end{tabular}

** rect: rectilinear; cyl: cylinder; irreg: irregular; alu: aluminium.

\section{TWO-COLOR LASER SCANNER}

The optical principle of this device is shown in Figure 3. The two laser beams are combined in a beamsplitter, the mixed eam is reflected towards the target by means of a small miror, concentrically located with the receiver optics. The canmirror makes 500 tiny steps over the scanline of $35^{\circ}$. Each step provides thus an angular increment of $1.2 \mathrm{mr}$. In each itep 3 measurements are carried out; laser 1 switches on, laser 2 switches on and both lasers off. These 3 events happen in $1.6 \mathrm{msec}$. The sample time is $3 \mu \mathrm{sec}$. The 500 samples per scanline are made in $800 \mathrm{msec}$. 


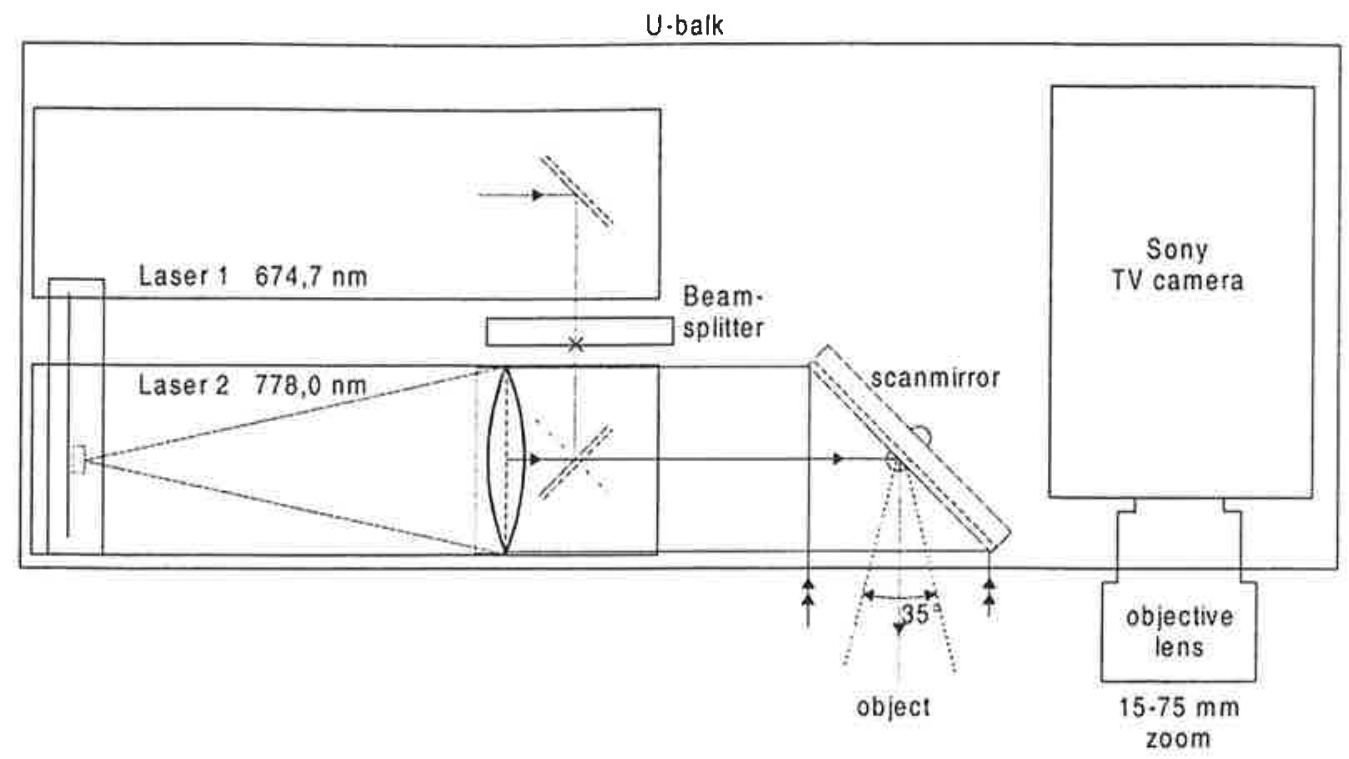

Figure 3: Optical principle of 2-color laser scanner.

Table 2: List of characteristics of two-color laser scanner.

\begin{tabular}{|l|l|}
\hline Parameter & Value \\
\hline wavelengths & 674.7 and $778 \mathrm{~nm}$ \\
laser power & about $5 \mathrm{~mW}$ \\
temperature stabilized & $20^{\circ} \mathrm{C}$ \\
receiver optics & $\mathrm{D}=40 \mathrm{~mm} \mathrm{f}=120 \mathrm{~mm}$ \\
detector & Siemens BPW $33 \quad 2.7 \times 2.7 \mathrm{~mm}$ \\
beam splitter & Melles Griot $03 \mathrm{BDS} 001(715 \mathrm{~nm})$ \\
detector spectral response & $($ Si) $500-1000 \mathrm{~nm}$ \\
responsivity & $2.5 \mathrm{~V} / \mathrm{Watt}$ \\
scan mirror & General Scanning, D = 50 mm, G350DT drive \\
scantime per line & $\sim 0.8 \mathrm{sec}$ \\
scanwidth at $2.7 \mathrm{~m}$ distance & $1.70 \mathrm{~m}$ \\
number of samples per line & 500 \\
data acquisition & $\mathrm{DT}$ labmaster $12 \mathrm{bits} \pm 10 \mathrm{~V} \ldots \pm 1.25 \mathrm{~V}$ \\
electronic bandwidth & $5 \mathrm{kHz}$ \\
noise level & $0.26 \mathrm{mV}$ RMS \\
feedback resistor & $4.3 \mathrm{M} \Omega$ \\
\hline
\end{tabular}

The data are acquired digitally. The black levels are subtracted from each retro-reflected laser signal. The signal strength B (bit level) follows from the formula:

$$
B=C^{\prime} \cdot W \cdot \frac{\rho}{\pi} \cdot \frac{\frac{\pi}{4} D^{2}}{R^{2}} \cdot \tau_{\text {opt }}=C \cdot \frac{\rho}{R^{2}} \text { from which } \rho=\frac{B \cdot R^{2}}{C}
$$

in which $\mathrm{W}$ is the laser power, $\rho$ the retro-reflection coefficient, $\mathrm{D}$ the optics diameter (entrance pupil), $\tau_{\mathrm{opt}}$ the optical transmission and $\mathrm{R}$ the range; $C^{\prime}$ and $\mathrm{C}$ are the intermediate resp final calibration constant. For the red and near IR laser the values for $C$ were obtained from the signal returns of a white labsphere plate: 1593 resp 1890. 

The software, controlling the mirror scans, allows oversampling per step up to 100 times. This may increase the signal to
noise ratio a factor 10 if required.

In the field experiments this was not done, as the signal to noise ratio was already adequate. In Figure 4 an example of a laser scanned image is shown in the evening of 31 October 2000 in wet conditions. The 3 images show the red, near IR and a mixed image, where the NDVI (Normalized Difference Vegetation Index $=\frac{\rho_{2}-\rho_{1}}{\rho_{2}+\rho_{1}}$ ) is presented.

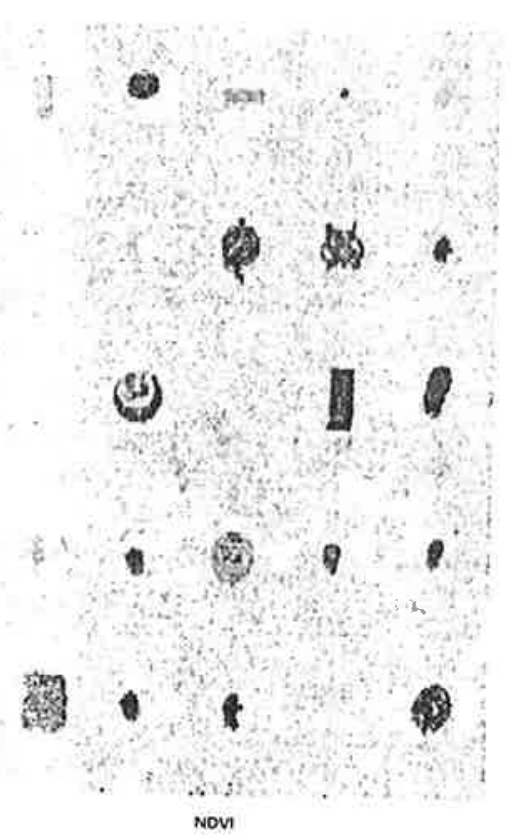

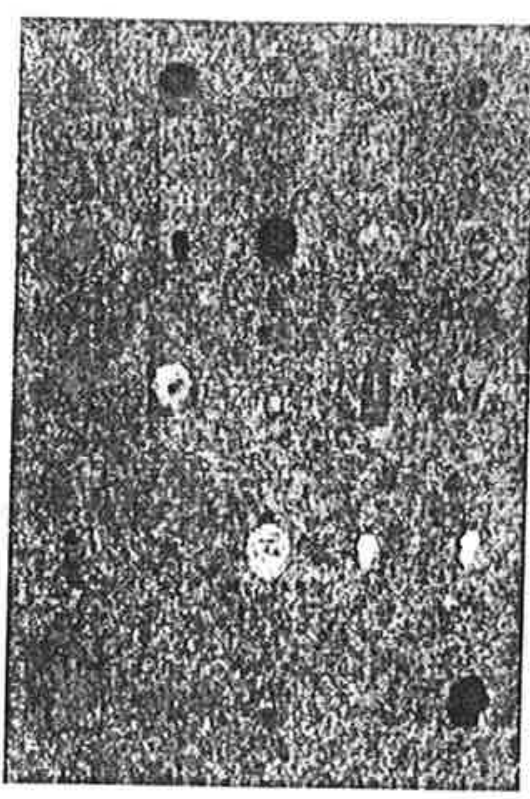

rod

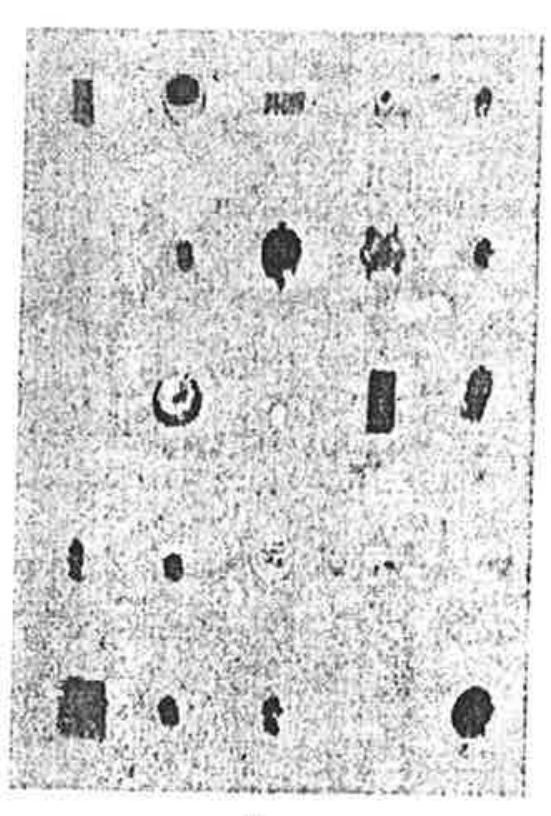

nit

Figure 4: Picture with laser scanner manual 31 October 2000; $675 \mathrm{~nm}$ (red), $778 \mathrm{~nm}$ (nir) and NDVI $=\frac{\text { nir-red }}{\text { nir }+ \text { red }}$. The distance sensor to target was $2.7 \mathrm{~m}$.

The data have been further analyzed and one example is shown in Table 3. Both the ratio $\rho_{1} / \rho_{2}$ and the clutter maps are presented for a number of objects from Table 1 . Most of the ratios correspond quite well with the ratios of Table 1 ; only for grass a different value is found due to the fact that the grass around the targets was more dead and yellow than the grass from Table 1. Striking, but expected is the difference in clutter from the objects and the grass.

Table 3: Field measurement ( 31 October) of selected objects; calibrated with white labsphere.

\begin{tabular}{|l|l|l|l|l|c|}
\hline Object & No & $\rho_{1}$ (red) & $\rho_{2}$ (nir) & $\rho_{1} / \rho_{2}$ & $\begin{array}{c}\text { RMS }\left(\rho_{2}\right) / \text { RMS } \\
\text { grass }\end{array}$ \\
\hline brown stone & 1A & 0.087 & 0.125 & 0.70 & 0.19 \\
wood (wet) & 1C & 0.131 & 0.193 & 0.68 & 0.38 \\
dark green disc & 2A & 0.116 & 0.40 & 0.29 & 0.20 \\
black rubber & 2C & 0.025 & 0.023 & 1.09 & 0.094 \\
green box & 3D & 0.109 & 0.097 & 1.12 & 0.11 \\
red/brown cyl & 3E & 0.150 & 0.131 & 1.145 & 0.10 \\
E31 cyl & 4B & 0.067 & 0.062 & 1.08 & 0.26 \\
brown UMWP & 4C & 0.30 & 0.41 & 0.73 & 0.36 \\
F16 PVC & 4D & 0.46 & 0.50 & 0.92 & 0.38 \\
red cylinder & 4E & 0.59 & 0.63 & 0.94 & 0.23 \\
concrete tile & 5A & 0.107 & 0.120 & 0.89 & 0.28 \\
grass between 4-5 & & 0.103 & 0.42 & 0.25 & 1.00 \\
\hline
\end{tabular}




\section{FOUR-CHANNEL SWIR RADIOMETER SCANNER}

The opto-mechanical principle of this device is shown in Figure 6. Two parallel optical channels provide four spectral bands by means of a manually variable filter switch. The filter characteristics are provided in Table 4, all operating in the Shor Wave IR (SWIR). In our experiments we have chosen to use only the two spectral bands $1596 \mathrm{~nm}$ and $2200 \mathrm{~nm}$. A summary with technical details on the SWIR radiometer scanner is provided in Table 5.

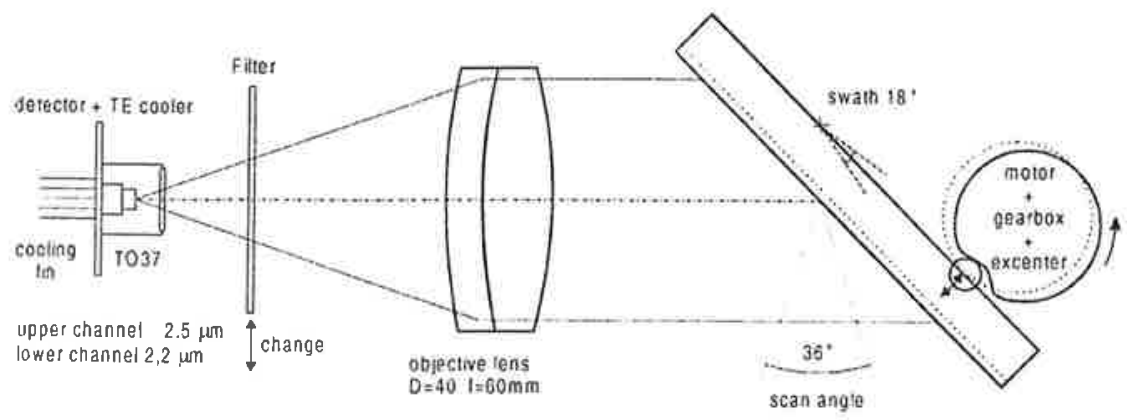

Figure 6: Opto-mechanical principle of 2-color SWIR radiometer scanner (top view, second channel below the first channel).

Table 4: Characteristics of filters in SWIR radiometer scanner.

\begin{tabular}{|l|c|c|c|}
\hline Detector & Center wavelength & 50\% bandwidth & Transmission \\
\hline SU2.2TE & $1482 \mathrm{~nm}$ & $24 \mathrm{~nm}$ & 0.41 \\
& 1596 & 35 & 0.45 \\
SU2.5TE & 2049 & 26 & 0.52 \\
& 2200 & 23 & 0.48 \\
\hline
\end{tabular}

The sensor design is based upon the use of the formula:

$$
E_{\lambda} \cdot \Delta \lambda_{f} \cdot \Delta \rho \cdot \frac{1}{4}\left(\frac{D}{f}\right)^{2} \cdot A_{d} \cdot \tau_{f} \cdot \tau_{0}=N E P \cdot \sqrt{\Delta f_{N}}
$$

in which $E_{\lambda}$ is terrain irradiance, $\Delta \lambda_{f}$ the spectral bandwidth of the optical filter, $\Delta \rho$ the minimum difference in reflection coefficient, $D$ and $f$ the lens diameter and focal length, $A_{d}$ the detector sensitive area and $\Delta f_{N}$ the noise bandwidth.

Technical data on the SWIR radiometer are given in Table 5. It is noted that the electrical bandwidth is not optimized with the scan speed. The signal to noise ratio can be $20 \times$ better. The detectors (from Sensors Unlimited) provide NEP values of about $0.6 \cdot 10^{-13} \mathrm{~W} / \mathrm{HHz}$. For a distance of $3 \mathrm{~m}$ we obtain a minimum detectable $\Delta \rho$ value of 0.006 (RMS) with $\mathrm{E}_{\lambda}=50 \mathrm{~W} / \mathrm{m}^{2} \mu \mathrm{m}$.

An example of an image in the field is shown in Figure 7; similar to the image of Figure 4 the targets were at a distance of $2.7 \mathrm{~m}$. The irradiance level, measured with the labsphere white panel, at $2200 \mathrm{~nm}$, was found to be $5.5 \mathrm{~W} / \mathrm{m}^{2} \mu \mathrm{m}$. The data were collected with the help of a Tektronix TDS3034 digital oscilloscope with $0.1 \mathrm{mV}$ resolution. The signals were corrected for the zero signal offset levels. 
Table 5: List of characteristics of two-color SWIR radiometer scanner.

\begin{tabular}{|l|l|}
\hline Parameter & Value \\
\hline wavelengths & $1596 \mathrm{~nm}(\mathrm{SU} 2.2 \mathrm{TE}) ; 2200 \mathrm{~nm}(\mathrm{SU} 2.5 \mathrm{TE})$ \\
detector size & $300 \mu \mathrm{m}$ diameter \\
operating temperature & $-30^{\circ} \mathrm{C}$ \\
receiving optics & $\mathrm{D}=40 \mathrm{~mm} \mathrm{f}=60 \mathrm{~mm}$ \\
noise level $\left(\Delta \mathrm{f}_{\mathrm{N}}=20 \mathrm{~Hz}\right)$ & $2 \mu \mathrm{V}(\mathrm{SU} 2.2 \mathrm{TE}) ; 6 \mu \mathrm{V}(\mathrm{SU} 2.5 \mathrm{TE})$ \\
broadband noise (RMS) & $200 \mu \mathrm{V}(\mathrm{SU} 2.2 \mathrm{TE}) ; 310 \mu \mathrm{V}(\mathrm{SU} 2.5 \mathrm{TE})\left(\Delta \mathrm{f}_{\mathrm{N}} \approx 100 \mathrm{kHz}\right)$ \\
feedback resistor & $6.6 \mathrm{M} \Omega$ \\
responsivity (no lens) & $7.64 \cdot 10^{6} \mathrm{~V} / \mathrm{W}(\mathrm{SU} 2.2 \mathrm{TE}, 1482 \mathrm{~nm}) ; 2.23 \cdot 10^{7} \mathrm{~V} / \mathrm{W}$ \\
& $(\mathrm{SU} 2.5 \mathrm{TE}, 2220 \mathrm{~nm})$ \\
point target responsivity (with lens) & $7.16 \cdot 10^{3} \mathrm{~V} / \mathrm{W} / \mathrm{m}^{2}(\mathrm{SU} 2.2 \mathrm{TE}, 1596 \mathrm{~nm}) ; 1.39 \cdot 10^{+} \mathrm{V} / \mathrm{W} / \mathrm{m}^{2}$ \\
& $(\mathrm{SU} 2.5 \mathrm{TE} .2220 \mathrm{~nm})$ \\
scan angle (total) & $36^{\circ}$ \\
no of IFOV's per line & 128 \\
time per scanline & $-0.8 \mathrm{sec}$ \\
data acquisition & Tektronix TDS3034 Digital oscilloscope; $0.1 \mathrm{mV}$ \\
no of samples per line & 500 \\
\hline
\end{tabular}

Table 6: Results of contrast measurements with SWIR scanner.

\begin{tabular}{|l|c|r|r|c|c|c|}
\hline \multirow{2}{*}{ Object } & \multirow{2}{*}{ No } & \multicolumn{2}{|c|}{ Bit level } & \multirow{2}{*}{$\rho_{1,6}-\rho_{\mathrm{gr}, 1.6} /$} & \multicolumn{2}{c|}{ RMS grass } \\
\cline { 3 - 4 } \cline { 7 - 7 } & & $1600 \mathrm{~nm}$ & $2200 \mathrm{~nm}$ & $\rho_{2.2-}-\rho_{\mathrm{gr}, 2.2}$ & $1600 \mathrm{~nm}$ & $2200 \mathrm{~nm}$ \\
\hline grass & & 100.5 & 87.5 & & 11.6 & 12.8 \\
brown stone & 1A & 128.8 & 129.6 & 0.67 & & \\
dark green disc & 2A & 91.1 & 152.3 & -0.15 & & \\
YMB4 & 3B & 116.1 & 100.5 & 1.20 & & \\
wooden box & 3D & 18.0 & 40.5 & 1.76 & & \\
UMWP & 4C & 191.3 & 125.4 & 2.40 & & \\
concrete tile top & 5A & 97.1 & 112.2 & -0.14 & & \\
concrete tile side & 5A & 126.0 & 128.6 & 0.62 & & \\
dark green cube & 5D & 110.4 & 181.7 & 0.11 & & \\
PPM2 black plastic & 5E & 12.1 & 41.9 & 1.94 & & \\
\hline
\end{tabular}

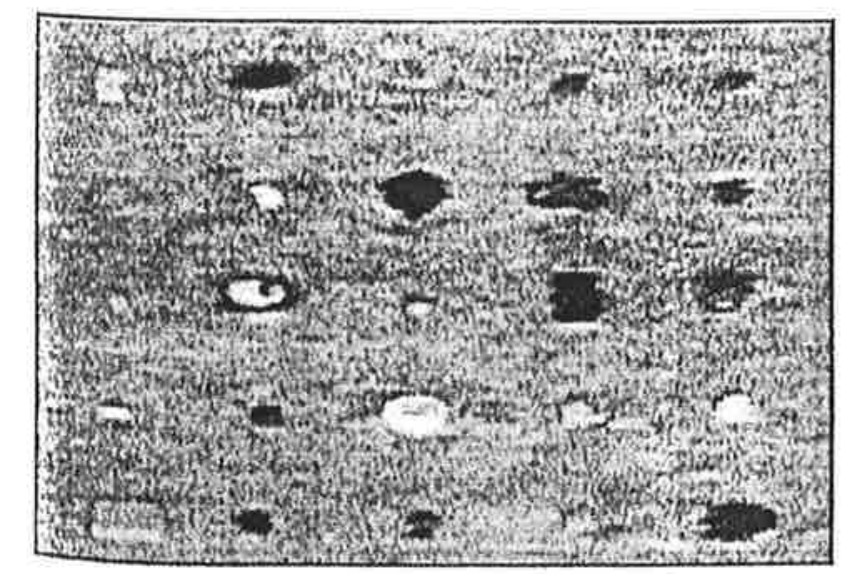

SWIR1600

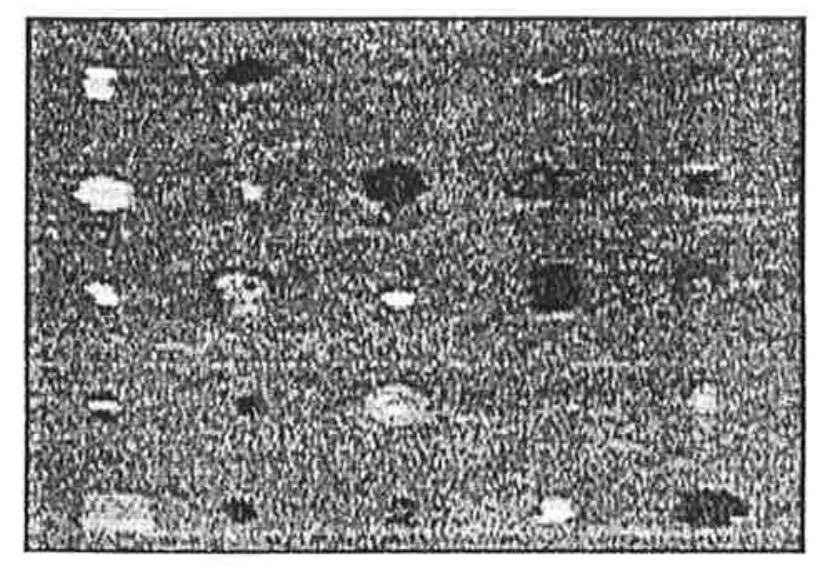

SWIR2200

Figure 7: Image of the minefield, taken with the SWIR scanner on 1 November 2000. 
The images of Figure 7 clearly show the differences in contrast of certain objects in both bands. This is also shown in Table 6 with a list of signal levels and contrast ratios, compared to grass. Most of the targets are clearly detected, although the signal to noise ratio is limited. Apparently the target illumination with the wide angle 500 Watt halogen lamps is not ideal; better is a line illumination source with cylinder optics. Also the spatial resolution is somewhat limited due to the $5 \mathrm{mr}$ instantaneous field of view of the detector. Figure 8 shows a raw signal plot across object line 5. The picture in Figure 7 shows that some objects have higher signals from the side parts than from the top, due to gloss of the wet top surface. Striking is the complementarity of the contrasts compared to the NDVI picture of Figure 4.

SWIR 2200 and $1600 \mathrm{~nm}$

1 nov 2000

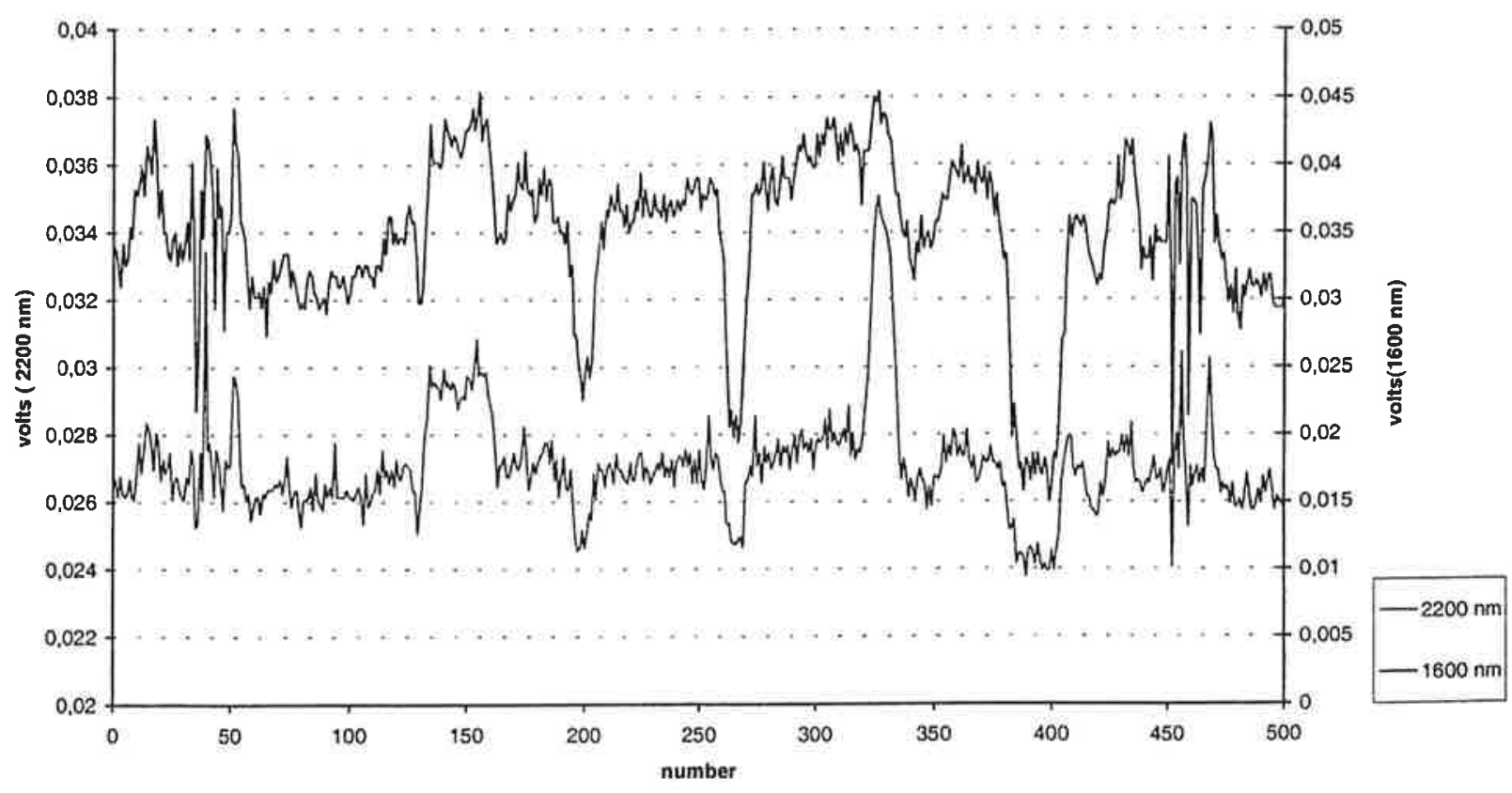

Figure 8: Two-color SWIR scanner signals over object line 5 (concrete tile to PPM2) including the edges of the lane, showing all 'mines'. The $1600 \mathrm{~nm}$ channel data were taken with an oscilloscope setting of $10 \mathrm{mV} / \mathrm{div}$; the $2200 \mathrm{~nm}$ channel with $5 \mathrm{mV} / \mathrm{div}$.

\section{MULTISPECTRAL CCD-CAMERA}

The major reason to incorporate a TV camera in a mine detection set is its spatial resolution. In the prefeasibility phase we used a Kodak camera with $1534 \times 1024$ detector elememts ([3]). In order to be more compatible with standard videorecorders, we decided however to select in this case a $1 / 2^{\prime \prime}$ CCD camera from Philips, providing a pixel size on the ground of less than $2 \mathrm{~mm}$. Technical data of the sensor are listed in Table 7.

As light sources were used the same set of wide beam halogen lamps, shown in Figure 2. The light distribution was not such that a homogeneous picture was obtained, but on one line the irradiance was sufficient. The sources were not designed for creating shadows, supporting shape recognition like in the setup of 1997 ([3]), where an array of flash lamps around the Kodak camera, individually flashing, was used. The filters consisted of a selection over the visual region at locations where optimum color discrimination is predicted.

An example of imagery in 6 spectral bands is shown in Figure 9. It is noted that the change in filters caused tiny displacements in the image, resulting in not-ideal colocation of pixels and impossibility to perform direct pixel to pixel correlation. Color discrimination is only possible by comparing bigger areas (more than $3 \times 3$ pixels) around each point. In doing so we can create a feature space with the restriction of the limited dynamic range of the CCD camera. 
Table 7: Characteristics of multispectral CCD camera.

\begin{tabular}{|l|l|}
\hline Parameter & Value \\
\hline number of pixels & $752(\mathrm{H}) \times 582(\mathrm{H})$ \\
chip dimensions & $6.4(\mathrm{H}) \times 4.8(\mathrm{~V}) \mathrm{mm}\left(1 \mathrm{r}^{\prime \prime}\right)$ \\
MTF & $0.80 @ 400 \mathrm{TV}$ lines $($ contour off $)$ \\
sensitivity & 0.25 lux $(\mathrm{f} / 1.0-6 \mathrm{~dB})$ \\
dynamic range & $59 \mathrm{~dB}$ \\
lens & computer $12.5-75 \mathrm{~mm} \mathrm{f} / 1.2$ \\
Field of View & $28.7^{\circ} \times 21.7^{\circ}-4.9^{\circ} \times 3.7^{\circ}(\mathrm{H} \times \mathrm{V})$ \\
spectral bands & $365,420,488,550,610,671,730$ and $800 \mathrm{~nm} ; 10 \mathrm{~nm}$ bandwidth \\
size & $50.8 \times 50.8 \mathrm{~mm}$ \\
\hline
\end{tabular}
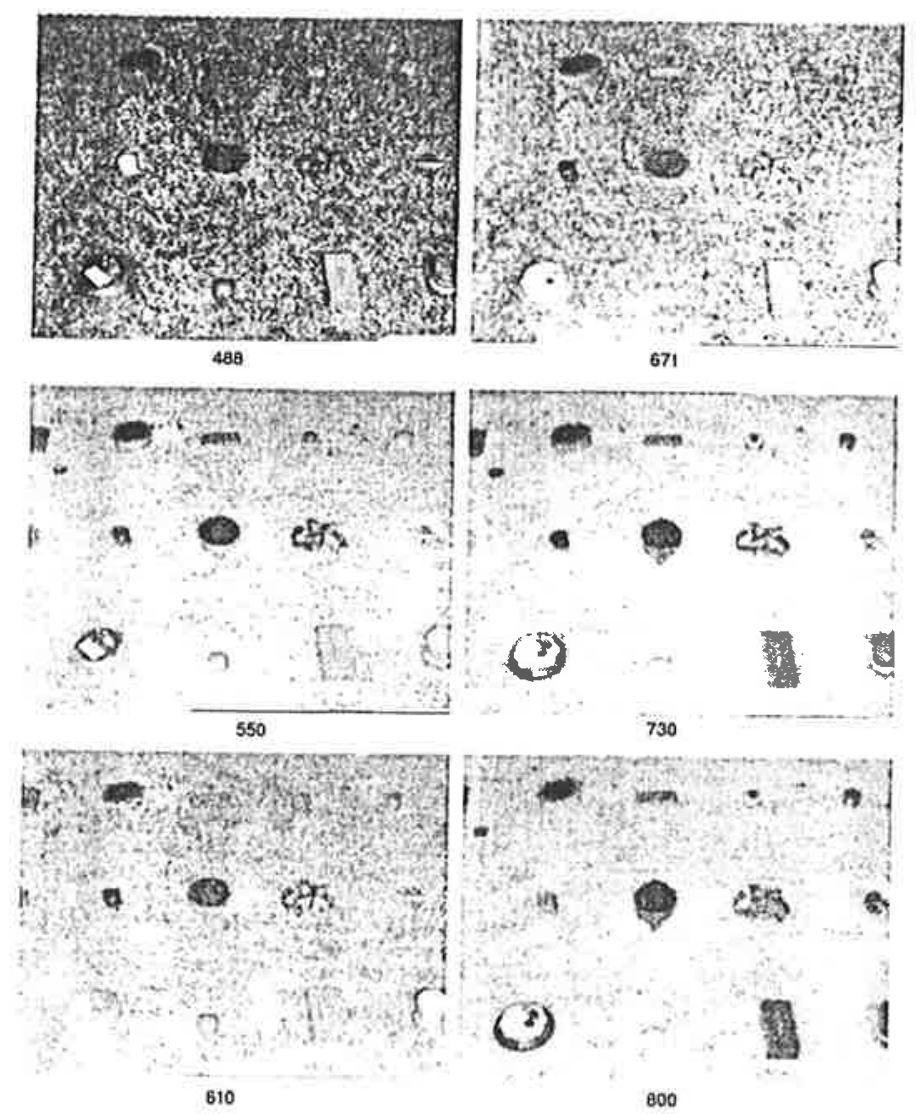

800

Figure 9: Images of lines 1, 2 and 3 in 6 spectral bands of the multispectral CCD camera; artificially illuminated with $4 \times 500$ Watt lamp set.

An example is shown in Figure 10, where contrasts: near IR $(800 \mathrm{~nm})-$ red $(671 \mathrm{~nm})$ are plotted against contrasts: red $671 \mathrm{~nm})$ - green $(550 \mathrm{~nm})$ for a number of targets including the target clutter area. The power of color discrimination for arget identification for a number of targets is clearly shown. It is also noted that the clutter in grass is much bigger, similar 0 the finding in the laser scanned image. 


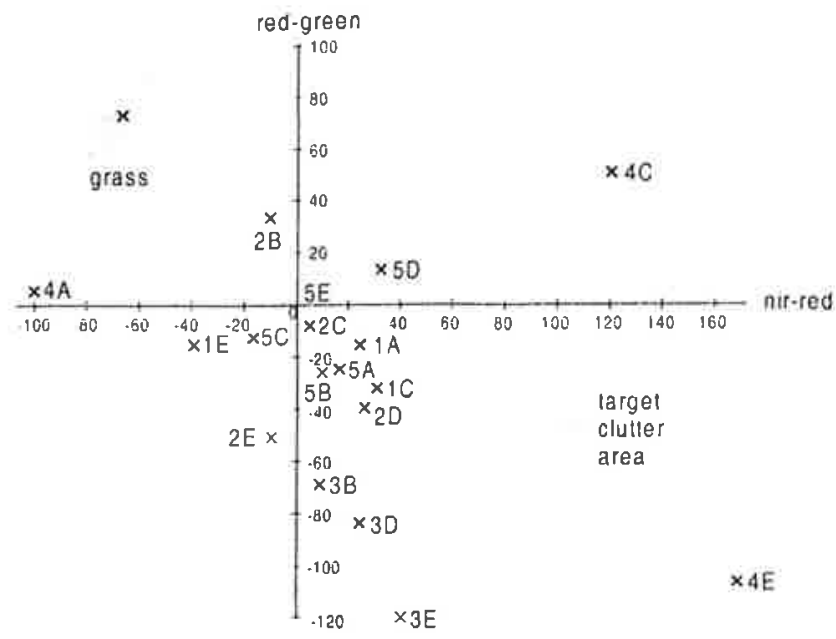

Figure 10: Multispectral radiance differences for various objects. Note that for each image black levels have been subtracted first.

The CCD camera was also used for gloss determination with polarization filters in front of the lamps (and camera) according to the setup, shown in Figure 11.

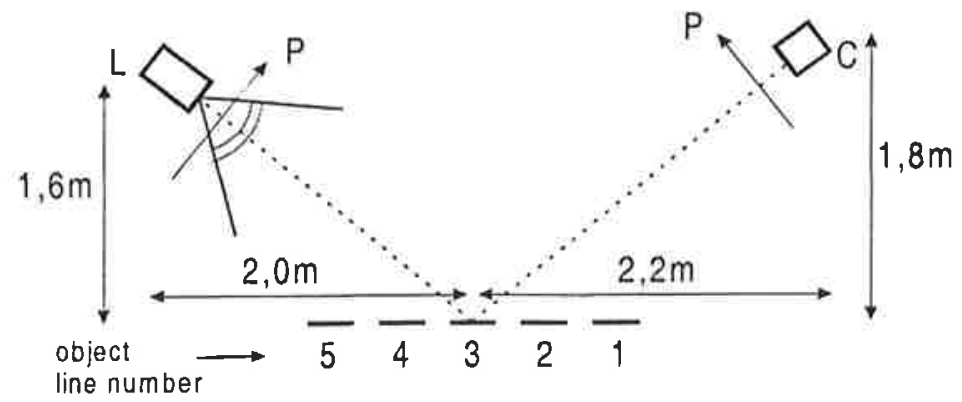

Figure 11: Schematic setup of polarization experiment, $L=4 \times 500 \mathrm{~W}$ lamps; $P=$ polarization filter; $C=C C D$ camera.

Because of the wet weather conditions, during most of the time gloss was present, as shown in Figure 12 (top image). The CCD camera was provided with a green filter in this case. The advantage of rotating the filter in front of the lamps is that there are no displacements of the image over the CCD pixels, allowing a simple image subtraction technique in a software package such as Paint Shop Pro. The bottom picture directly provides $100 \%$ target detection and shape/size target identification for more than $50 \%$ of the targets. 


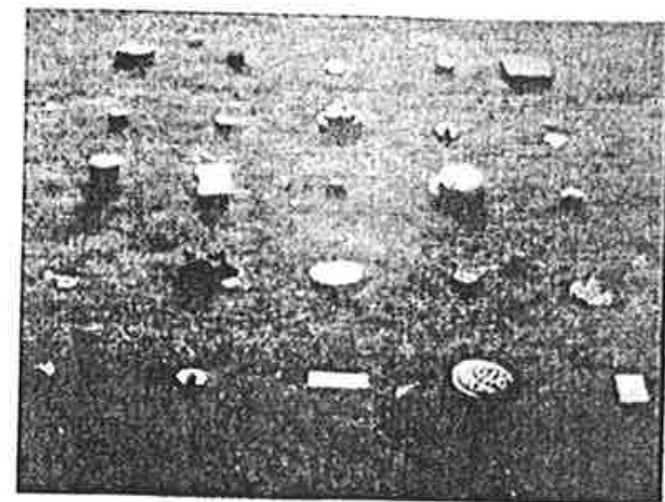

pol 4

camera $\rightarrow$

lamp $\rightarrow$

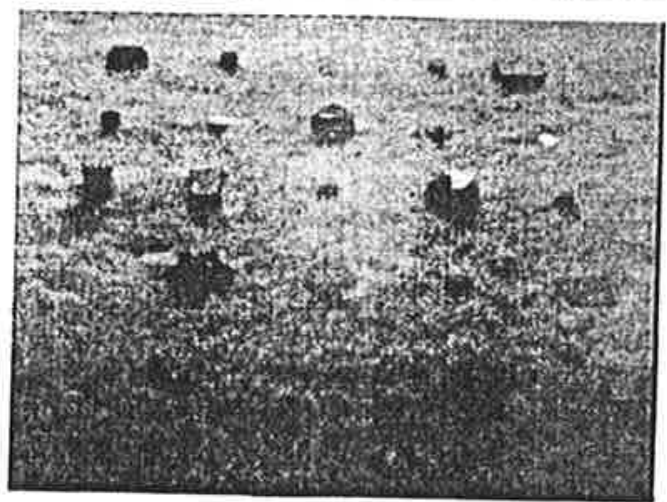

pol 1

camera $\rightarrow$

lamp $\uparrow$

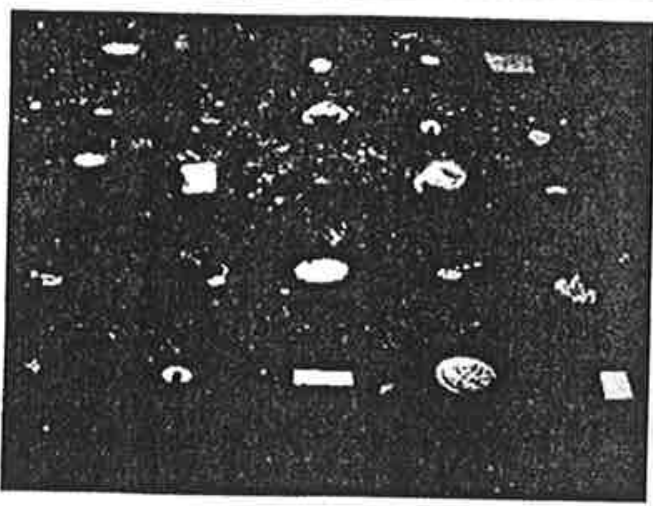

diff 4-1

Figure 12: CCD camera images with various polaroid positions for the lamps; bottom image is difference image between top and middle image.

\section{DISCUSSION}

The experiments with the active multispectral sensors have shown the power of discrimination when using a variety of target and background features, as indicated in Table 8. It is clear that the combined sensor package is capable to provide near $100 \%$ identification probability for the 25 objects presented in Table 1 . The most difficult objects are numbers $1 \mathrm{D}$ and $4 \mathrm{~B}$, which have the same shape and size and the same top color; the only difference exists on the side part of the cylinder. 
Table 8: Success and presence of discrimination features from active multispectral sensors.

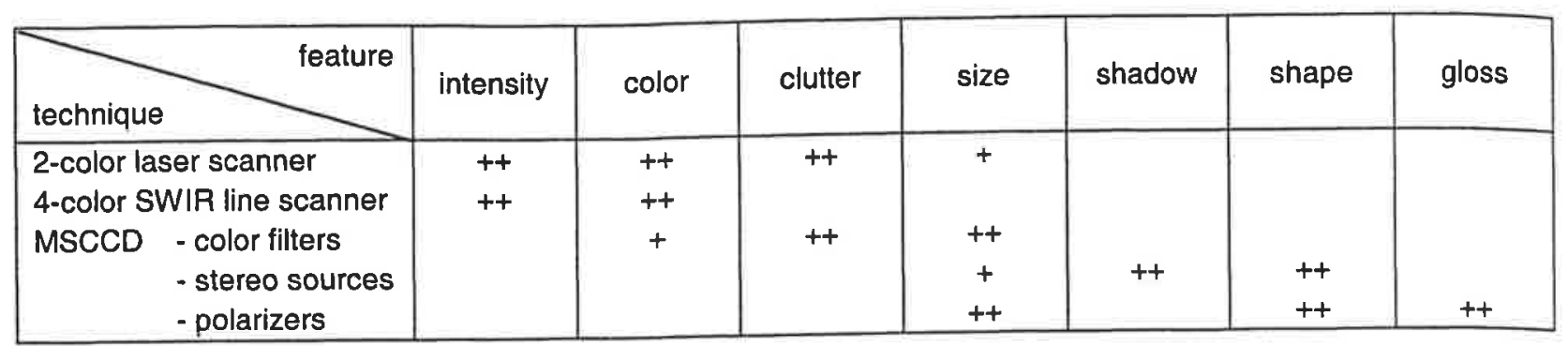

The situation of near perfect identification changes of course when real surface mines, partly overgrown by vegetation or partly covered with soil or dust, have to be detected. Based upon the experience, described in this paper, it is advised to use the active techniques, combined with a kind of waterspray system to clean the area under investigation. There remains however work to be done to optimize the sensor package and the processing algorithms and to enhace the speed of the detection system.

\section{REFERENCES}

[1] Arie N. de Jong, Study on feasibility of automatic identification and discrimination based on size, color and temperature, TNO report PHL 1970-23 (in Dutch)

[2] K.M. Lee, Shape from shading with a Generalized Reflectance Map Model, Computer Vision and Image Understanding, Vol 67 No. 2, August 1997

[3] Arie N. de Jong, Testing Active Mine Detection Setups, TNO report FEL-97-A370 (in Dutch)

[4] Wim de Jong et al., Experiments with alternative mine detection methods, FEL-99-A105 (in Dutch)

[5] Wim de Jong et al., Sophisticated test facility to detect landmines, Proc SPIE, Vol 3710, Orlando, April 1999

[6] Arie N. de Jong et al., Experiments with active Multispectral EO Mine Detection Sensors, TNO report FEL-01-A001, January 2001 